


\title{
Adsorption Mechanisms in Layer-by-Layer Films
}

\author{
Maria Raposo ${ }^{1,2}$ and Osvaldo N. Oliveira $\mathrm{Jr}^{1}$ \\ ${ }^{1}$ Instituto de Fúsica de São Carlos, \\ Universidade de São Paulo, \\ CP 369 - 13560-970 São Carlos, SP, Brazil \\ FAX: +55 16 2713616, e-mail: [raposo,chu]@ifsc.sc.usp.br \\ ${ }^{2}$ Departamento de Física, \\ Faculdade de Ciências e Tecnologia, \\ Universidade Nova de Lisboa, 2825 Monte Caparica, Portugal
}

Received 08 September, 1998

\begin{abstract}
Adsorption processes in layer-by-layer films are discussed using poly(o-methoxyaniline) (POMA) as a case study and also comparing with results for other polymers from the literature. This follows a brief overview of the materials and characterization techniques employed for self-assembled films, including their possible applications. The original paradigm of the self-assembly method is associated with spontaneous adsorption of oppositely charged polymer layers. While this rationale has been successful in explaining adsorption mechanisms for some polyelectrolytes, for polyanilines other interactions must be included. For POMA, in particular, at least three types of interactions are identified, namely van der Waals forces, ionic interactions and $\mathrm{H}$-bonding. Furthermore, $\mathrm{H}$-bonding is responsible for a number of effects even for charged POMA where electrostatic attraction was expected to predominate. Such effects include POMA dedoping upon contact with a glass substrate at early stages of adsorption, and the non-linear increase in the adsorbed amount with the number of POMA/poly(ethenesulfonic acid) (PVS) bilayers deposited in a multilayer structure. Adsorption of a POMA layer on a glass substrate or on an already formed POMA/PVS film occurs in two steps: a fast, first-order kinetics process with a characteristic time of a few seconds and a slower process represented by a Johnson-Mehl-Avrami function with a characteristic time of hundreds of seconds. These correspond basically to nucleation and growth mechanisms which is corroborated by atomic force microscopy measurements. The amount of material adsorbed in any given layer depends on experimental parameters, especially polymer concentration and $\mathrm{pH}$, owing to the different extents of $\mathrm{H}$-bonding that may allow POMA to adsorb on itself or on PVS molecules.
\end{abstract}

\section{Introduction}

The search for novel materials with molecular control has prompted worldwide research on organic materials that offer a variety of electrical, optical and magnetic properties to be exploited in molecules based devices and sensors. The Langmuir-Blodgett (LB) technique $[1,2,3,4]$ has been perhaps the most extensively employed for such purposes, in particular because it allows multilayer architectures to be produced with controllable thickness at the nanometer level and high degree of order depending on the organic material used. The self-assembly (SA) methods [2,3] appeared as an alternative to the LB technique, providing a means to build multilayer structures with considerably less sophisticated experimental procedures since they are based on the spontaneous adsorption of the material of interest on a substrate or on an already deposited layer. The first of such methods to be exploited was self-assembly via chemisorption [2], which allows very stable multilayer films to be produced, but is obviously restricted by requiring chemical reactivity of the molecules end groups for successful adsorption. In 1991, Decher [5,6,7] proposed a self-assembly method that makes use of electrostatic interaction of oppositely charged layers. This rationale had in fact been used be- 
fore by Nicolau [8] for growing polycrystalline epitaxial thin films of metals via the alternating adsorption of cations and anions from solutions containing the respective salts $[8,9]$, and also for building layer-by-layer films of polypyrrole and polyaniline polymerized in situ [10]. Decher applied this approach to polyelectrolytes, in which alternating layers of polycations and polyanions were spontaneously adsorbed on a solid substrate, with the resulting films resembling LB films as far as the molecular architecture and thickness control was concerned. Rubner [11-14] was among the first to realize the potential of Decher's approach and extended the method to conjugated polymers. A tremendous upsurge of interest in this self-assembly (SA) method followed which is now used for building multilayers of a large variety of materials, stemming from polymers [11-18], to proteins [19-22]. Furthermore, it has been demonstrated [17] that interactions other than the electrostatic attraction can be exploited in building self-assembled films, thus opening up new possibilities of molecular architectures. For example, Shimazaki et al [23] prepared layer-by-layer films based on the charge-transfer (CT) interaction between electrondonating carbazolyl groups and electron-accepting 3,5dinitrobenzoyel groups in the side chains of two kinds of methacrylate polymers [23].

The immense potential for applications has driven the vast majority of research work to be concentrated on the fabrication and characterization of selfassembled films that display the properties of interest. For instance, SA films have been successfully used in producing light emitting diodes (LEDs) [24,25] from poly(p-phenylene vinylene) (PPV) for which the molecular engineering possibilities have been essential. The electroluminescence can be enhanced by choosing adequate materials to be alternated with PPV $[24,25]$. Less attention has been paid to the physics of the adsorption processes involved in building the multilayers. It is on this topic that we have focused our work. We first started $[15,18]$ by producing SA films of poly(o-methoxyaniline) (POMA), a polyaniline derivative, alternated with polyanions such as sulphonated polystyrene (SPS) and poly(ethene sulfonic acid) (PVS), with the aim of investigating the electrochromic properties of POMA. While searching for optimized deposition conditions, we noted however that the film properties could vary substantially if the experimental conditions were varied. This prompted us to extend investigations to the adsorption processes, including kinetics of adsorption, substrate dependency and interaction energies involved [18,26-28]. The main findings of this 4-year research project are reported here.

The paper is organized as follows. In Section II an overview is presented of the materials that have been reported for building SA films, also including the techniques for film characterization and possible applications of such films. Section III discusses the buildup of multilayer self-assembled films, with emphasis on substrate effects that must be considered while analyzing film properties. The mechanisms of adsorption are discussed in Section IV, where we also elaborate on the importance of $\mathrm{H}$-bonding as the driving force for the adsorption of polyaniline self-assembled films.

\section{Self-assembly (SA) or layer -by-layer films}

A brief overview of the recent literature on selfassembled (SA) films is presented. For more detailed information the reader is referred to excellent reviews that can be found in ref. [3,4]. As stated in the Introduction, the search for new materials for device applications has been a strong motivation in the development of fabrication methods. The need to build multilayers arises because the adsorption of a single layer hardly reaches the required device thickness, of the order of 500 to $2000 \mathrm{~nm}$ [28]. For a long time the LangmuirBlodgett (LB) technique was the only one to provide the required thickness and architecture control while building organic multilayers. The self-assembly (SA) method appeared as an alternative, initially based on the chemical adsorption of organic molecules with specific functional groups, (see, for example [2,29-32]). In order to obtain multilayers it was necessary to chemically adsorb a layer onto an already deposited layer, which required the synthesis of molecules with adequate affinity. This is by no means a straightforward task, which then imposes a severe limitation in the use of this self-assembly method. Decher's approach [5-7] circumvented this problem, by producing films out of the electrostatic attraction between oppositely charged lay- 
ers, i.e. by adsorbing alternatively anionic and cationic polyelectrolytes on solid supports [5-7]. The formation of these multilayers is based on the tendency of pairs of oppositely charged polyelectrolytes to form complexes [33]. An advantage of this technique is that the adsorption processes are independent of the substrate size and topology, with film thickness increasing linearly with the number of adsorbed bilayers [5-7]. Another advan- tage lies in the possibility of building up heterostructures with different materials with a specified functionality. The potential of this method may be illustrated by the wide range of materials that have been employed, such as polyelectrolytes [5-7,33,34], polymers [11-18, 24, $25,35]$, polymers containing dyes [36-39], biological materials [19-22], and ceramics [40,41]. A summary of several materials used is shown in Tables I and II.

Table I. Polycations used in the buildup of layer-by-layer films

\begin{tabular}{|l|c|c|}
\hline \hline POLYCATIONS & ACRONYM & REFERENCES \\
\hline$\alpha, \beta, \gamma, \delta$-tetrakis(1-N-methylpyridyl) & TMPyP & 36 \\
porphine tetrakis(p-toluenesulfonate) & - & 19 \\
Calf thymus type YIII-S histone & Lys & 19,20 \\
Chicken egg white lysozyme & Cyt c. & 19,20 \\
Cytochrome c from horse heart & Eu(III) & 46 \\
Europium & Mb & 19,20 \\
Horse heart myoglobin & Hb & 20 \\
Horse hemoglobin & MV & 36 \\
Methylviologen & Per & 20 \\
Peroxidase & PAA & 49 \\
Poly(allylamine) & PAH & $6,13,19,34,43,50,54,56$ \\
Poly(allylaminehydrochloride) & PAN or PANi & 35,56 \\
Polyaniline & PEI & $19,20,21,36,37,40,43$ \\
Poly(ethylenimine) & PDDA & $19,20,37,40,43$ \\
Poly(diallyldimethylammonium) & PL & 54 \\
Poly(L-lysine hydrobromide) & PVP & 54 \\
Poly(2-vinylpyridine) & PMA & 33 \\
Poly(dimethylamino) ethylmethacrylate & PDAC & 38,47 \\
Poly(dimethyldiallylammonium chloride) & PMPA & 13 \\
Poly[(N-methyl-pyridinium-2-yl)acetylene] & PPV precursor & $13,24,25$ \\
Poly(p-phenylenevinylene) precursor & PPY & 14 \\
Polypyrrole & POEA & 16 \\
Poly(o-ethoxyaniline) & POMA & $16,26-28$ \\
Poly(o-methoxyaniline) & PVI+ & 33 \\
Partially quaternized polyvinylimidazole & & \\
\hline \hline
\end{tabular}


Table II. Polyanions used in the buildup of layer-by-layer films.

\begin{tabular}{|l|c|c|}
\hline \hline POLYANIONS & ACRONYM & REFERENCES \\
\hline Acid Red 26 & AR26 & 36 \\
Acid Red 27 & AR27 & 36 \\
Ammonium octamolybdate & $\left(\mathrm{NH}_{4}\right)_{4}\left[\mathrm{Mo}_{8} \mathrm{O}_{26}\right]$ & 43 \\
Bacteriorhodopsin & BR & 38 \\
Bovine liver catalase & Cat & 20 \\
Concanavalin A Canavalia Ensiformis & Con A & 20 \\
Congo Red & LR & 36 \\
Diaphorase & DIA & 20 \\
Ferritin & FER & 21 \\
Glucoamylase Aspergillus niger & GA & 19,20 \\
Glucose oxidase & GOD & $19,20,21$ \\
Indigo Carmine & IC & 36 \\
Poly(acrylic acid) & PAA & 33,56 \\
Poly(anilinepropanesulfonic acid) & PAPSA & 19 \\
Poly(ethenesulfonic acid) & PVS & $16,26-28$ \\
Polyimide precursor & PMA & 13 \\
Poly(methacrylic acid) & PTAA & $24,25,33,56$ \\
Poly(thiophene-3-acetic acid) & SPS or PSS & $12,13,19,20,21$, \\
Poly(styrene sulfonate) & & $24,25,33,34,36,37$, \\
& & $40,43,47,49,54,56$ \\
Poly(1-(4-(3-carboxi-4-hydroxyphenylazo)- & PAZO & 7 \\
benzenesulphonamido)-1,2-ethanediyl) & & \\
Poly[2-(3-thienyl)ethanol hidroxy- & H-PURET & 46 \\
carbonyl-methylmethane] & PM & 38 \\
Purple membrane & SPAn & 13 \\
Sulfonated polyaniline & & \\
\hline \hline
\end{tabular}

An equally wide variety of experimental techniques have been employed in the analysis of SA film properties. Perhaps the most extensively used is UV-visiblenear-infrared spectroscopy since most candidate materials for the SA method absorbs light in this wavelength region, and this spectroscopy provides a direct means of estimating the amount of material adsorbed and also the multilayer buildup. The multilayer thickness can be measured by profilometry, ellipsometry and atomic force measurements. Photoluminescence and electroluminescence have also been measured for the SA films made from luminescent polymers such as PPV [24,25]. The order of SA films has been probed by neutron reflectivity measurements [42] and small angle X-ray reflectivity (SAXR) [6], as will be commented upon later. The quartz crystal microbalance has been used for obtaining the adsorbed amount which is determined from the frequency shift of the quartz resonator and using the
Sauerbrey equation [35,43]. Atomic force microscopy (AFM) [18,21,38], scanning angle reflectometry [44], second harmonic generation $[38,39]$ and thermally stimulated desorption [27] have also been employed for investigating SA film properties.

As for the possible applications of SA films, the first extensive efforts have been made by Rubner's group in the fabrication of light emitting diodes (LEDs) employing PPV initially [24,25] and then ruthenium II complexes ( $\mathrm{Ru}$ (bpy) $3^{2+}$ polyester) [45]. Polymer light emitting diodes were also prepared with poly[2-(3thienyl)ethanol hidroxycarbonyl-methyl methane] (HPURET) and europium (Eu(III)) layer-by-layer films [46]. Similarly to other thin solid films, layer-by-layer films may be applied to sensors, integrated optics, friction reducing coating and surface orientation layers. Clark et al [47], for instance, discovered a process of patterning ionic layer-by-layer films with micron-sized 
features, exploiting the ability of reverting the polyelectrolyte deposition on the self-assembled monolayer surfaces at very high salt concentration, thus creating a negative to the original positive structure. This may lead to fabrication of more complex structures, and then applied to optical or electrical devices [47].

\section{Multilayer buildup}

In the layer-by-layer or self-assembly (SA) method, the substrate is immersed in a beaker containing either a polycationic or polyanionic solution for a given period of time (usually a few minutes). The substrate is then washed in a solution of approximately the same $\mathrm{pH}$ of the polymeric solutions, in order to remove nonadsorbed molecules, and usually dried as well. The substrate+film system is now charged which allows adsorption of an oppositely charged layer upon immersion in the other polymeric solution. Following adsorption of this second layer, the substrate is again washed and dried. Multilayer structures can then be built by repeating the steps above as long as required. Apparently, there are no limitations in the number of bilayers that can be deposited; films with hundreds of layers have been reported. Even though data from the literature will be used in illustrations and comparisons, we shall base our analysis on results with the POMA/PVS films. The experimental procedures adopted for producing such SA films have been described in ref. [18], and therefore only a short description is given here. POMA was synthesized with ammonium peroxydisulfate in a 1.0 $\mathrm{M} \mathrm{HCl}$ aqueous solution at $0^{\circ} \mathrm{C}$. The monomer to oxidant ratio was 4:1. The resulting precipitated polymer was filtered and washed with a $1.0 \mathrm{M} \mathrm{HCl} \mathrm{solution.}$ POMA has a weight average molecular weight $\left(\mathrm{M}_{w}\right)$ of $30,800 \mathrm{~g} / \mathrm{mol}$ [48]. Poly(ethenesulfonic acid) (PVS) was purchased from Aldrich Chemical. These polymers are, respectively, cationic and anionic at low pHs, and therefore multilayer structures can be built via electrostatic interaction of alternating layers.

An important feature of layer-by-layer films is the linear increase in thickness or in the adsorbed amount per unit area with the number of bilayers. Fig. 1 shows the dependence with the number of bilayers of both total thickness, calculated from the distance be- tween two neighboring Kiessig fringes from small angle X-ray scattering data, and the optical absorbance at $225 \mathrm{~nm}$, for polystyrenesulfonate and polyallylamine layer-by-layer films [5]. It was noted that the thickness and the absorbance do not increase linearly from zero, as one might have expected. Indeed, it is now well established that substrate effects are important for the first few layers, leading to a non-linear build up in the initial stages of adsorption. We have analyzed this feature very carefully for the POMA/PVS system [18]. Fig. 2 shows the buildup of POMA/PVS films on several substrates [18]. Glass I and glass II refer to glass substrates that have been submitted to different preparation procedures prior to adsorption. It can been seen that deviations from the linear behavior occurred for the first few layers, especially for the Teflon FEP substrate. The latter presented the least stable SA films owing to lack of adhesion; in addition, the strong deviation from the linear behavior may be due to the time of adsorption allowed for each layer. It is possible that the $3 \mathrm{~min}$. employed was far below the necessary for Teflon. In a subsequent investigation for POMA/PVS on glass, the amount of adsorbed POMA was found to increase exponentially in the first few layers and only increase linearly after the sixth or seventh bilayer [28]. This was attributed to weak attractive interactions between the substrate and the polymer, which could be demonstrated by the shift in the UV-vis. absorption peak as the adsorption of the first POMA layer proceeded (Fig. 3). The interaction with the substrate caused POMA to be dedoped thus shifting the absorption curve to lower wavelengths due to a mechanism involving $\mathrm{H}$-bonding as will be discussed in Section IV. The increase in the amount of adsorbed POMA with the number of layers is explained by the increase in adsorption sites when some polymer is already adsorbed. Such an observation was confirmed in atomic force microscopy (AFM) measurements that showed an increase in film roughness as additional layers are adsorbed. Fig. 4 shows that the mean roughness increases for the first few bilayers, until reaching a constant value, in much the same way as the amount of POMA adsorbed per layer [28]. 


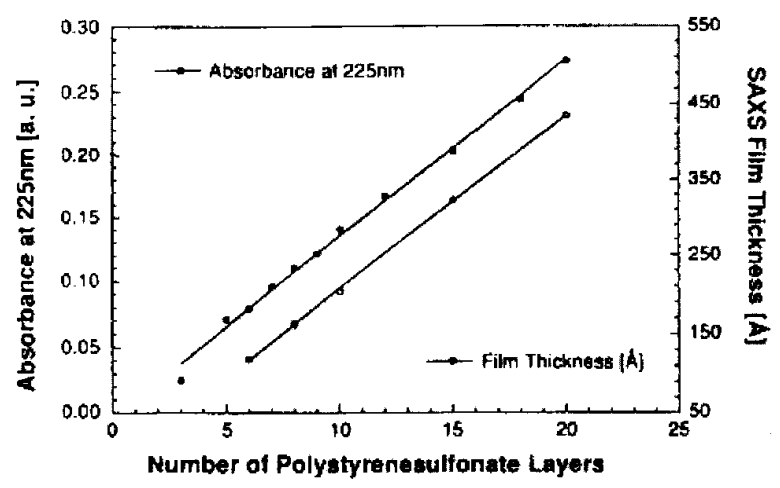

Figure 1. Total thickness and the optical absorbance at $225 \mathrm{~nm}$ of layer-by-layer films of polystyrenesulfonate and polyallylamine adsorbed onto a fused quartz as a function of the number of polystyrenesulfonate layers [5].

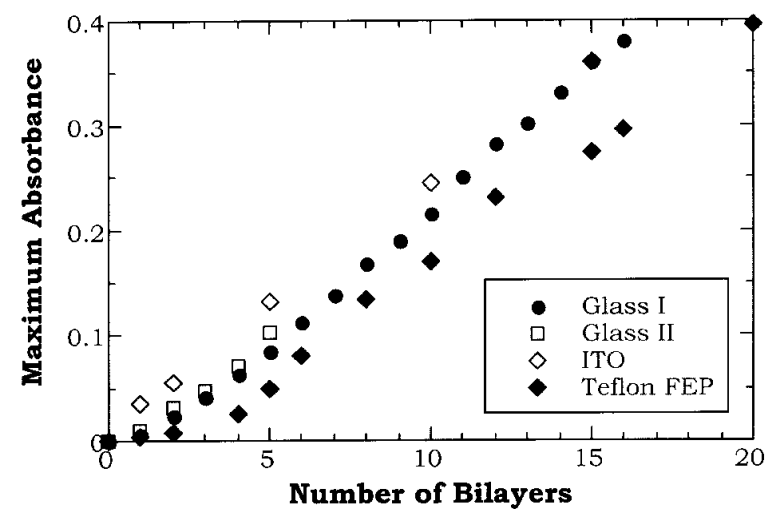

Figure 2. Maximum absorbance versus number of bilayers of POMA/PVS films adsorbed on: Teflon FEP, ITO, glass I and glass II [18].

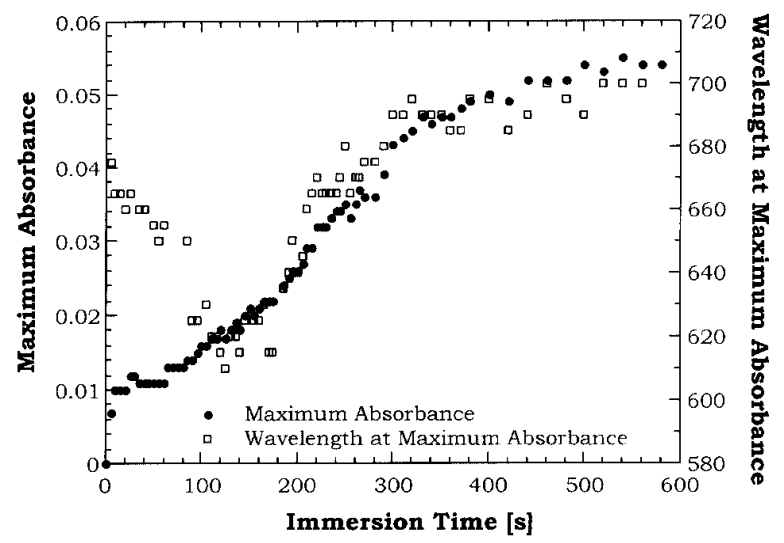

Figure 3. Absorbance and wavelength at maximum absorbance of adsorbed POMA on hydrophilized glass as a function of immersion time [18].

An opposite behavior was observed in layer-bylayer films from poly(o-methoxyaniline) alternated with poly(thiophene acetic acid) (POMA/PTAA). Interestingly, the amount of material adsorbed is very large in the first bilayers but then it becomes constant, leading to a linear increase from the fourth layer (2 bilayers) on, as shown in Fig. 5. The reason for this lies in the strong attractive interaction between the glass substrate and PTAA molecules. Similar results where obtained by Ferreira et al [11] in a similar polymer system. Owing to the importance of substrate effects for the first few bilayers, results in the literature now tend to fall in these categories of non-linear buildup, with either a superlinear or a sublinear increase in the amount of material adsorbed with the number of layers. A linear increase from zero has become scarce.

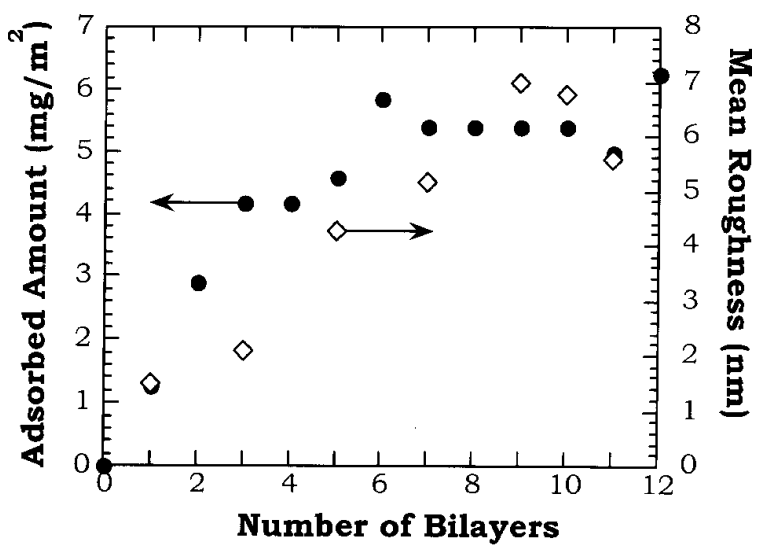

Figure 4. The adsorbed amount and mean roughness of POMA/PVS SA films versus the number of bilayers [28].

The buildup of multilayers has so far been discussed with no mention to the time allowed for adsorption, whether it was sufficient for the formation of a complete layer or not. In fact, a linear build up may be observed even when the time allowed is not enough for saturation of the adsorption process for a given layer. This occurs provided that the period of adsorption is maintained constant for all layers, as illustrated in Fig. 6 for self-assembled films of ammonium octamolybdate $\left(\left(\mathrm{NH}_{4}\right)_{4}\left[\mathrm{Mo}_{8} \mathrm{O}_{26}\right]\right)$ and poly(allylaminehydrochloride) $(\mathrm{PAH})$ [43]. The amount of adsorbed material was estimated using a quartz crystal microbalance whose frequency shift was plotted against the immersion time. The time period of adsorption of $\left(\mathrm{NH}_{4}\right)_{4}\left[\mathrm{Mo}_{8} \mathrm{O}_{26}\right]$ is varied for the three series of experiments as indicated in the figure, with $\mathrm{PAH}$ being adsorbed for 15 minutes in all experiments [43]. A similar behavior was found for POMA/PVS films where saturation in the adsorption process takes approximately 2 hours, but a linear build up (after few bilayers) of the 
multilayer structure may be obtained using a constant immersion time of three minutes for each layer.

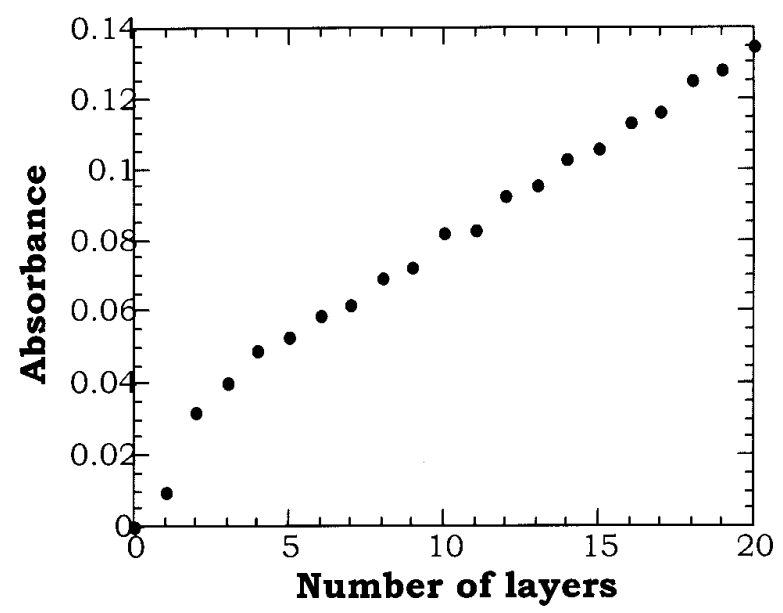

Figure 5. Maximum absorption (at $430 \mathrm{~nm}$ ) as a function of the number of POMA/PTAA bilayers. Here both POMA and PTAA absorb at $430 \mathrm{~nm}$.

The layer-by-layer method was conceived with the aim of fabricating organized structures. This is probably more likely to be achieved in the perpendicular direction to the substrate, owing to the layered structure $[5,6,7]$. Nevertheless, some ordering in the plane may also be obtained if adequate materials are employed, as demonstrated by Lvov [37] for polymers with side chain nonlinear optical dyes. They obtained orientation of chromophore side-chain groups of the polymer poly[1-[4-(3-carboxyl-4-hydroxyphenylazo)benzene sulfonamide]-1,2-ethenediyl, Na salt] (PAZO) alternated with poly(dimethyldiallylammonium chloride) (PDDA), which was confirmed by second harmonic generation (SHG) measurements. A maximum of orientation was obtained for 4 PDDA/PAZO bilayers. Another example of molecular organization was obtained by $\mathrm{He}$ et al [38] who produced layer-by-layer films of purple membrane (PM), isolated from Halobacterial halobium R1M1, and poly(dimethyldiallylaminonium chloride) (PDAC). Using second harmonic generation, the latter authors measured the second-order susceptibility of PDAC/PM layer-by-layer films (12 bilayers) and obtained a larger value compared with electrophoretically sedimented films [38].

\section{Adsorption processes}

Adsorption is the final result of the competi- tion among several interactions that include polymer/substrate, polymer/polymer, polymer/solvent and solvent/substrate interactions. Furthermore, polymer molecules may adopt a completely different conformation when adsorbed as compared to that in solution. Consequently, as far as experimental conditions are concerned, the adsorption process depends on the concentration, $\mathrm{pH}$ and ionic strength of the polymeric solutions, type of substrate and immersion time. No theory has been developed specifically for SA films, and the complexity of the molecules involved and the dependency on so many parameters make it difficult to adapt existing adsorption theories. One may nevertheless analyze separate aspects of adsorption in a phenomenological way in order to gain insights into the physical mechanisms involved. This is precisely what we have tried to do, focusing on the POMA/PVS system, though other systems from the literature are also analyzed for comparison. We first investigated the kinetics of adsorption of the first layer on a bare substrate and then of an nth layer on an already deposited film. By varying the polymer concentration and carrying out experiments at different temperatures, we could obtain adsorption isotherms which may allow estimation of the thermodynamic energies of the system. This is done, however, by using extremely simplified models of adsorption while interpreting the data. The limitations of such analysis are commented upon in Section 4.2. Finally, attempts have been made to distinguish between electrostatic interactions and hydrogen bonding as the driving force for adsorption of polyaniline multilayers.

\section{1- Kinetics of adsorption}

The immersion time required for adsorption of a given layer in SA multilayer structures is the first experimental condition to be identified whenever a new polymeric system is introduced. Full coverage of the substrate for the first layer, for instance, may take distinct immersion periods depending on the substrate and also on the material to be adsorbed. The amount of material adsorbed is also dependent on the immersion time. From the point of view of the physical mechanisms, establishing the kinetics of adsorption is obviously illustrative of the types of interaction that prevail in the 
adsorption processes. The first systematic investigation of kinetics in SA films is due to Ferreira and Rubner [13] who carried out a number of experiments with various concentrations and types of substrate for the poly(thiofene-3-acetic acid) (PTAA) and PAH system. Their data for adsorption of PTAA on 5 PTAA/PAH bilayers deposited on a positively charged substrate are shown in Figure 7 for various PTAA solution concentrations. Light absorption was measured at $420 \mathrm{~nm}$ [13], corresponding to the absorption peak for PTAA. These results reveal that PTAA has a high affinity for the filmcovered substrate since the adsorbed amount increases rapidly with time. After the first minute of immersion the adsorbed amount is $72 \%, 78 \%$ and $80 \%$ of the final adsorbed amount value, respectively for concentrations of $10^{-4} \mathrm{M}, 10^{-3} \mathrm{M}$ and $10^{-2} \mathrm{M}$. These results show also that the concentration plays an important role, a point that will be considered later on. Their results were interpreted as being due to a diffusion-controlled adsorption process, at least in the initial stages of adsorption. However, the effective diffusion coefficients varied widely with the concentration, which made Ferreira and Rubner [13] conclude that the Langmuir-Schaefer relationship is not valid for PTAA in the time regime accessible by UV-vis experiments.

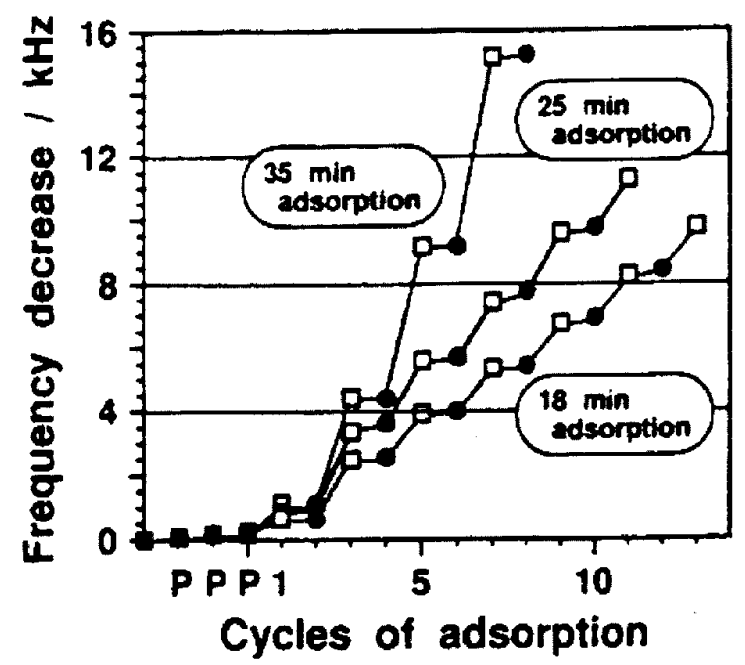

Figure 6. Frequency shift in a quartz crystal microbalance as a function of adsorption time: (open square) $\left(\mathrm{NH}_{4}\right)_{4}\left[\mathrm{Mo}_{8} \mathrm{O}_{26}\right]$ adsorption; (full circle) $\mathrm{PAH}$ adsorption [43].

In the initial investigation by Ferreira and Rubner [13] there was no concern in distinguishing between the kinetics of adsorption of the first layer and that of a subsequent layer. Such a distinction is made here for the POMA/PVS system, since our experiments indicated a strong dependence on substrate effects. Fig. 8 shows the kinetics curves for the first POMA layer on bare hydrophilized glass substrates. An interesting effect was observed when the number of interruptions for washing the film and taking the UV-vis. absorption measurement was varied. In curve I the substrate was immersed in the polymeric solution for a few times and the absorbance of the sample was measured. In curve II the time of each immersion was $5 \mathrm{~s}$, and therefore a much larger number of points were obtained. In curve III several substrates were used and each one was immersed only once in the polymer solution. Note that in curve III the concentration of POMA solution was 0.35 $\mathrm{g} / \mathrm{L}$ while for curves I and II the concentration was about $0.8 \mathrm{~g} / \mathrm{L}$. The adsorbed amount changes drastically with the number of immersions. Therefore, one may conclude that the "true" kinetics of adsorption must be obtained by immersing the substrate only once in the polymeric solution, as in curve III. In spite of the differences in the adsorbed amount, the data of all three curves in Fig. 8 can be fitted by the following empirical equation [18]:

$$
A=k_{1}\left(1-\exp \left(-\frac{t}{\tau_{1}}\right)\right)+k_{2}\left(1-\exp \left(-\frac{t}{\tau_{2}}\right)^{n}\right)
$$

where $A$ is the absorbance at the peak which is proportional to the POMA adsorbed amount per unit area, $k_{1}$ and $k_{2}$ are constants, $\tau_{1}$ and $\tau_{2}$ are the characteristic times and $\mathrm{n}$ is a constant. Therefore, POMA adsorption may be attributed to two types of process: an initial, fast first order kinetics with characteristic times of a few seconds, followed by a much slower process that is fitted with a Johnson-Mehl-Avrami function with much higher characteristic times (hundreds of seconds). The latter function is generally believed to occur in processes of diffusion; when $1.5<\mathrm{n}<2.5$ the process occurs with a diffusion-controlled growth of domains and decreasing nucleation rate. For $n=1.5$ the process is due to diffusion controlled growth of domains and zero nucleation rate. Then, the first process would correspond to nucleation while in the second process there is only growth of domains. This hypothesis was corroborated by atomic force microscopy (AFM) measurements [18], which showed that within a few seconds of immersion, 
nuclei appeared all over the sample, and for longer immersion times larger domains were observed.

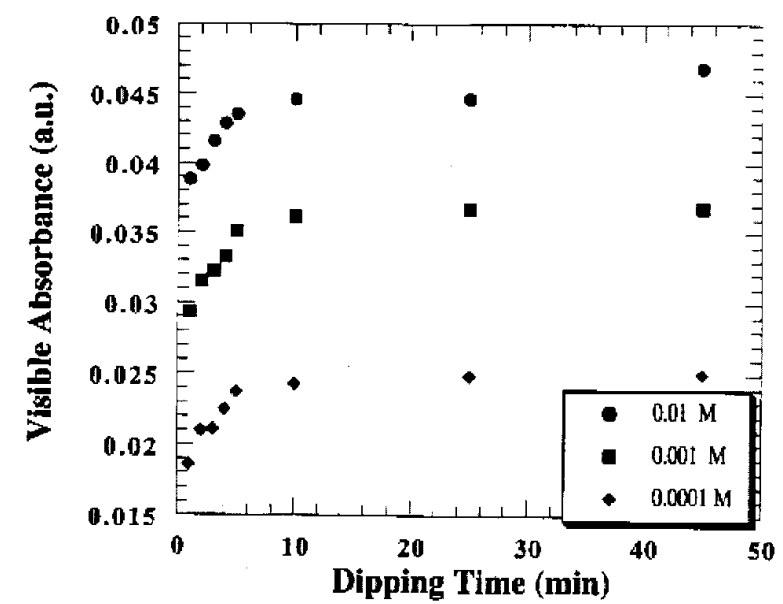

Figure 7. Kinetics of adsorption of PTAA onto 5 bilayers of PTAA/PAH for various concentrations. The absorbance was measured at $420 \mathrm{~nm}$ [13].

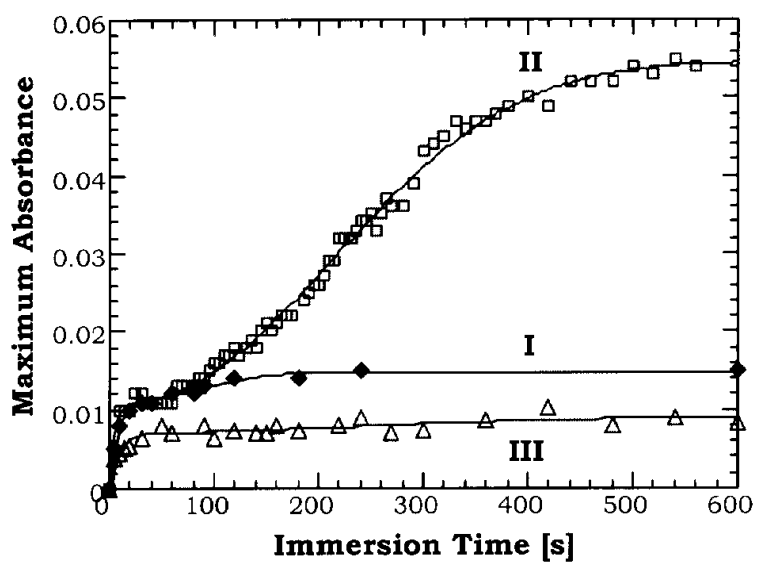

Figure 8. Kinetics of adsorption of poly(o-methoxyaniline) onto a glass substrate: I- Few points were taken using only one substrate. II- Points were taken at every $5 \mathrm{~s}$ using only one substrate. III- Several substrates, immersed only once for distinct periods of time.

As for the kinetics of a POMA layer onto an already formed POMA/PVS film, it was originally thought [18] that adsorption would become faster as the number of bilayers increased. However, this conclusion was based on experiments in which the substrate was immersed several times in the polymeric solution for the data points to be obtained. When the true kinetics was measured, i.e. with substrates immersed only once in the polymeric solution for that given layer, we noticed that the kinetics of adsorption of the nth layer also followed Eq. (1) [18]. The only major difference was that the characteristic time for the second process (domain growth) was much longer because a larger quantity of material was adsorbed in the nth layer, as will be discussed later.

A two-stage adsorption process was also proposed by Tsukruk et al [49] for layer-by-layer films of poly(styrenesulfonate) (PSS) and poly(allylamine) (PAA). They based this proposal on surface morphology changes. During the first stage (1-2 minutes) of film formation the polymer chains are inhomogeneously adsorbed on selected sites of the substrate (in this case an oppositely charged self-assembled monolayer), which had a high local charge concentration as in scratches, holes, edges and foreign microparticles. At the second stage, for longer deposition times (10-30 minutes) the polymer islands are gradually spread out and the roughness decreases. This feature was interpreted as caused by the high charge concentration in macromolecular chains [49]. In the case of POMA/PVS samples the sample roughness increased with the number of bilayers, tending to a constant value [28]. Even though Ferreira and Rubner [13] did not mention the possibility of a two-stage kinetics process, a close inspection in their data (Figure 7) points to a small plateau starting at approximately 2 minutes of adsorption, which could also be interpreted as the appearance of a second adsorption process.

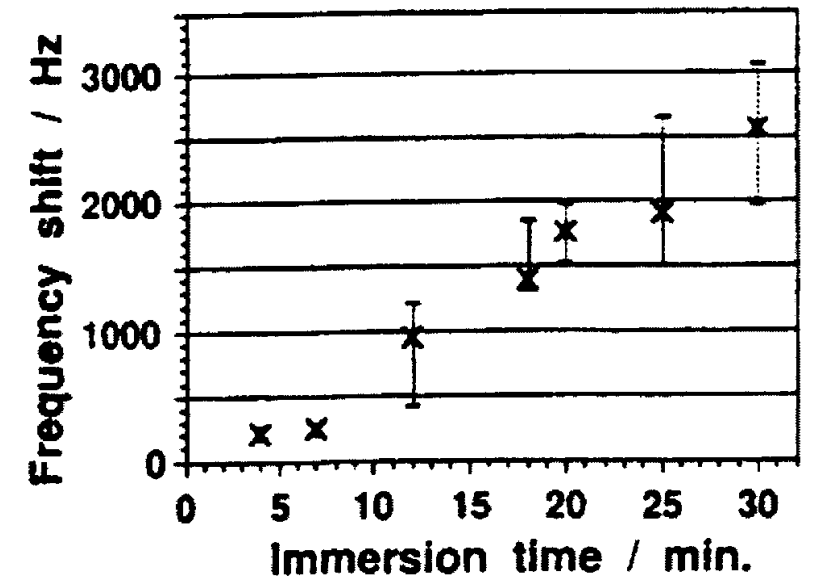

Figure 9. Variation of the quartz crystal microbalance frequency in the $\left.\mathrm{NH}_{4}\right)_{4}\left[\mathrm{Mo}_{8} \mathrm{O}_{26}\right]$ adsorption. Each point is an average frequency shift of four to six repeated adsorption processes within one series of experiments. [43].

There are reports in the literature, however, in which only one stage is observed for adsorption. For example, Ichinose et al [43] studied the kinetics of adsorption of ammonium octamolybdate $\left(\left(\mathrm{NH}_{4}\right)_{4}\left[\mathrm{Mo}_{8} \mathrm{O}_{26}\right]\right.$ 
onto films of $\left(\mathrm{NH}_{4}\right)_{4} \mathrm{Mo}_{8} \mathrm{O}_{26} / \mathrm{PAH}$ by plotting the quartz crystal microbalance frequency shift as a function of the immersion time as shown in Fig. 9. In contrast to the examples above no saturation occurred for the observed period of time (30 minutes).

\subsection{Adsorption Isotherms}

The concentration of the polymeric solution is an important parameter to be controlled in the fabrication of SA films. By varying the concentration, one may obtain the so-called adsorption isotherms such as those shown for POMA onto glass in Fig. 10 adsorbed at 25 and $40^{\circ} \mathrm{C}$ [26].The increase in the adsorbed amount at $25^{\circ} \mathrm{C}$ for concentrations higher than 0.25 $\mathrm{g} / \mathrm{L}$ was attributed to the increase of POMA aggregation in the aqueous solution. For concentrations lower than $0.25 \mathrm{~g} / \mathrm{L}$ the data can be fitted with a Langmuir isotherm, from which thermodynamic energies are estimated. The adsorption energy was $\sim-35 \mathrm{~kJ} / \mathrm{mol}$, the enthalpy of adsorption was $20 \pm 2 \mathrm{~kJ} / \mathrm{mol}$, while the entropy of adsorption was $183 \mathrm{~J} / \mathrm{Kmol}$. From these values one could conclude that POMA adsorption onto glass is controlled by entropy, that is, the increase in entropy in the solvent makes the polymer adsorption favorable, in spite of the positive enthalpy. It must be stressed that a positive adsorption enthalpy does not mean that the interactions between the substrate and the solute are repulsive. It simply means that energy must be supplied for adsorption to occur. By analyzing substrate effects we concluded that such energy is spent in breaking hydrogen bonds between substrate sites and water molecules that are immediately adsorbed upon contact of the substrate with the aqueous solution [28]. One of the consequences of these substrate effects is that the polymer adsorbed in the initial stages is less doped than in solution, as shown by the shift in the absorption peak depicted in Fig. 3. POMA at $\mathrm{pH}=3$ is $50 \%$ positively charged and has an absorbance peak at about $720 \mathrm{~nm}$. Upon losing some of its charge the peak shifts towards lower wavelengths. The shift occurs during the nucleation phase, but the peak returns to higher wavelengths after $200 \mathrm{~s}$ of immersion time, when the polymer being adsorbed probably does not interact with the substrate directly.

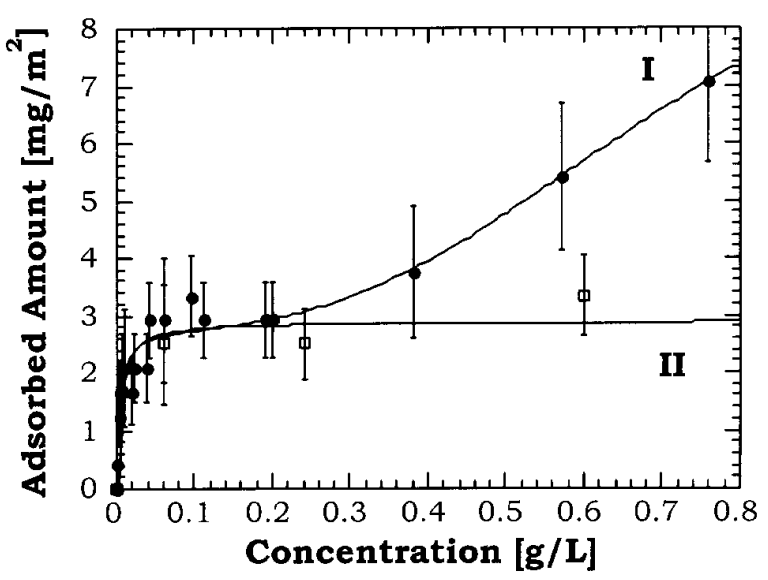

Figure 10. Adsorption isotherms of poly(o-methoxyaniline) onto glass at different temperatures:I- $25^{\circ} \mathrm{C}$ and II- $40^{\circ} \mathrm{C}$ [26].

A word of caution is due with regard to the use of a Langmuir isotherm in the interpretation of adsorption data for POMA. The simple model leading to the Langmuir isotherm was developed for adsorption of noninteracting hard spheres of negligible size. Obviously, the POMA system does not fulfill any of these premises. Moreover, POMA is a heterodisperse polymer which could be the reason why round isotherms such as that of Fig. 10 are observed [50]. In this case, the area per volume ratio should also influence the isotherm, but for the POMA experiments this ratio was rather low and therefore the isotherms were still expected to be round. Since experimental conditions such as the extent of POMA aggregation in solution could affect the shape and temperature dependence, some authors take the view [51] that the energy values extracted from the fittings with Langmuir isotherms are meaningless. Owing to the lack of theoretical models that can be applied to complex systems such as heterodisperse polymers, a number of other authors still employ the Langmuir isotherm fittings in order to get estimates of the energies involved [52]. In our work [26], in particular, such analysis allowed us to identify the substrate influence on the adsorption process. For an opposite trend in the temperature dependence was observed for the adsorption isotherms when an nth POMA layer was deposited on an already formed POMA/PVS film, with smaller adsorbed amounts at higher temperatures and low concentrations. In contrast to the first POMA layer, a negative enthalpy was obtained which demonstrates that substrate effects are no longer important and that adsorption on sites containing some polymer is favored, 
consistent with the increase in the amount of adsorbed material with the number of bilayers deposited. Nevertheless, before independent calorimetric measurements of enthalpy are made, experimenters should be aware of the strong reservations expressed by Fleer [51] in the analysis, even if phenomenological, of their adsorption data.

\subsection{Mechanisms for polymer adsorption}

Adsorption of any given layer depends on energetic factors such as electrostatic forces, van der Waals forces, hydrogen bonds, solvent quality, and entropic factors. Analogously to the analysis of adsorption isotherms mentioned above, the identification of the mechanisms responsible for polymer adsorption is also a complex task because theoretical models are at an embrionic stage as far as heterodisperse polymers are concerned. Several models have been proposed, mainly for polyelectrolytes, but they usually require knowledge of experimental parameters that is not available. For instance, even though the enthalpy of adsorption can be measured directly using calorimetric methods, the entropy and the free energy of adsorption cannot be measured easily. Additional difficulties are associated with the large variety of experimental conditions that affect the adsorption processes. Each trio of solute-solventsubstrate represents a new case, and in layer-by-layer films it is necessary to investigate the processes for at least two materials that form the alternating layers.

A phenomenological analysis may be successful, however, if one manages to distinguish between the various types of interaction. For instance, in the selfassembled (SA) films, whose paradigm is based on the electrostatic attraction of oppositely charged layers, electrostatic interactions are expected to predominate. Using the surface force apparatus, Lowack and Helm [53] investigated the solute/substrate interactions between negatively charged PSS onto a positively charged PAH monolayer, when they noted that only one third to one half of the positive charges are neutralized by PSS molecules. These results were interpreted via a Cohen-Stuart model [53] which states that polyelectrolyte molecules adsorb only until they are electrostatically repelled from the surface and not until the binding places are saturated, which would correspond to a "true equilibrium". The Cohen-Stuart model also justifies the difficulty of removing adsorbed polyelectrolytes from a surface under adverse conditions such as during the washing process. Indeed, no desorption is observed when POMA/PVS layer-by-layer films are placed in aqueous solutions for several days at room temperature. Hoogeveen et [33] showed that the multilayer stability is largely determined by both the electrostatic interactions of the anionic-cationic polymer pair and the polymer charge densities. They concluded that when the polymer with a very low charge $(<5 \%)$ is adsorbed onto a surface, practically all polymer charges are bonded to the substrate. Only few remain available for further adsorption, so that no adsorption of the next layer occurs as the attraction between polymer molecules is too weak. When the polymer charge is between $5-20 \%$ the next layer may be formed but the conformation of the first layer will somewhat adjust itself to the new layer. In some cases, during such adjustment bonds between the first layer and the substrate are broken, with both layers being desorbed. When the polymer charge is even higher $(>20 \%)$ the bonds between polymer-substrate and polymer layers are strong, and stable multilayers can be formed [33].

Another adsorption mechanism was proposed for amphoteric polymers by Kaniyama and Israelachvili [54] in which the number of discrete ionic bonds that can be formed between the charged groups on the polymer and on the surface plays an important role. In summary, adsorption would be determined by the details of the charge distribution and not simply by the average charge of the polymer and the surface, and therefore adsorption is assumed to be dependent on short-range electrostatic (coulombic) interactions rather than on a long-range or continuum-type interaction.

For the POMA/PVS system we also expected the electrostatic interactions to predominate. However, polyanilines are extremely prone to H-bonding, as pointed out by Stockton and Rubner [17] who suggested that hydrogen bonding was the driving force for adsorption of polyanilines in layer-by-layer films. Also, Benjamin et al [55] produced layer-by-layer films via hydrogen bonding between the amine group of poly(ethylleneimine) and the hydroxy group of the co(phydroxyV-PV) conjugated polymer. We have confirmed the importance of $\mathrm{H}$-bonding by investigating 
the POMA/PVS system at $\mathrm{pH}=3.0$ where POMA is protonated (i.e. charged) and also at higher pHs where POMA is neutral. Even at $\mathrm{pH}=3.0$, thermally stimulated desorption data showed three types of interaction in the POMA/PVS multilayers, namely van der Waals forces, hydrogen bonds and ionic interactions [27]. The presence of H-bonding was confirmed by FTIR spectroscopy of such films. That H-bonding was essential for POMA adsorption was demonstrated by the $\mathrm{pH}$ dependence of the adsorbed amount. For the first layer as well as for the nth POMA layer the maximum adsorbed amount occurred for a $p H \cong 6$, at which POMA is completely dedoped, and therefore ionic attraction is vanishing. The ability of polyaniline molecules to form H-bonds among themselves, in addition to the PVS molecules, also explains why the amount of adsorbed POMA increases with the number of layers already deposited [28]. The probability of adsorption increases in sites where some polymer is already adsorbed, which also causes the roughness to increase with the number of layers, as observed by AFM measurements. This is in contrast to the results of Lowack and Helm [53] where defects and roughness of surface polyelectrolyte multilayer films were found to be unimportant for the adsorption processes. As mentioned before, in their films the electrostatic attraction was the predominant factor.

\section{Conclusions}

Adsorption processes for the fabrication of layer-bylayer films have been analyzed, using POMA as a case study but also discussing data from other polymers. It was shown that $\mathrm{H}$-bonding plays a fundamental role in the adsorption of polyanilines on a glass substrate or on a PVS layer, even when the polymers were charged and electrostatic attraction was expected to predominate. The implications for adsorption kinetics and adsorption isotherms were discussed. Because several types of interaction may contribute to adsorption, the fabrication of SA usually depends on a number of experimental parameters that may be varied for the required molecular control. While this dependence represents a rich variety of possibilities for applications, it also calls for more systematic studies in the fundamental physics of the processes involved.

\section{Acknowledgements}

The authors acknowledge the financial support from FAPESP and CNPq (Brazil) and FCT - "SubPrograma Ciência e Tecnologia do $2^{\circ}$ Quadro Comunitário de Apoio" (Portugal). They are also thankful to Dr. Ernesto C. Pereira for providing them with the PTAA polymer.

\section{References}

[1] G.G. Roberts, Langmuir-Blodgett films, (PlenumPress, 1990).

[2] A. Ulman, An introduction to ultrathin organic films: from Langmuir-Blodgett to self-assembly, (Academic Press, 1991).

[3] R. H. Tredgold, Order in thin organic films, (Cambridge University Press, 1994).

[4] M. C. Petty, Langmuir-Blodgett films: an introduction, (Cambridge University Press 1996).

[5] G. Decher, J.D. Hong and J. Schmitt, Thin Solid Films, 210/211, 831 (1992).

[6] Y. Lvov, H. Haas, G. Decher and H. Möhwald, J. Phys. Chem. 97, 12835 (1993).

[7] Y. Lvov, G. Decher, H. Möhwald, Langmuir 9, 481 (1993).

[8] Y. F. Nicolau, Application of Surface Science 22/23, 1061, (1985)

[9] Y. F. Nicolau and J.C. Menard, J. of Crystal Growth 92, 128 (1988).

[10] Y. F. Nicolau and M. Nechtschain in Electronic Properties of Conjugated Polymers III, Springer Series in Solid-State Sciences, Vol 91, edited by H. Kuzmany, M. Mehring and S. Roth (Springer-Verlag Berlin, Heidelberg, 1989) p. 461.

[11] M. Ferreira, J.H. Cheung and M.F. Rubner, Thin Solid Films 244, 806 (1994).

[12] J. H. Cheung, A.F. Fou and M. F. Rubner, Thin Solid Films 244, 985 (1994).

[13] M. Ferreira and M.F. Rubner, Macromolecules 28, 7107 (1995).

[14] A.F. Fou and M.F. Rubner, Macromolecules 28, 7115 (1995).

[15] L.H.C. Mattoso, V. Zucolloto, L.G. Patterno, R. van Griethuijsen, M. Ferreira, S.P. Campana and O. N. Oliveira Jr, Synth. Met. 71, 2037 (1995).

[16] L.H.C. Mattoso, L.G. Patterno, S.P Campana and O.N. Oliveira Jr., Synth. Met. 84, 123 (1997).

[17] W.B. Stockton and M.F. Rubner, Macromolecules 30, 2717 (1997). 
[18] M. Raposo, R.S. Pontes, L.H.C. Mattoso and O.N.Oliveira Jr., Macromolecules 30, 6095 (1997).

[19] Y. Lvov, K. Ariga, I. Ichinose and T. Kunitake, J. Am. Chem. Soc. 117, 6117 (1995).

[20] Y. Lvov, K. Ariga, I. Ichinose and T. Kunitake, Thin Solid Films 284, 797 (1996).

[21] M. Onda, Y. Lvov, K. Ariga and T. Kunitake, Jpn. J. Appl. Phys. 36 L1608 (1997).

[22] C.E. Borato, P.S.P. Herrmann, L.A. Colnago, O.N. Oliveira Jr., L.H.C. Mattoso, Braz. J. Chem. Eng. 14, 367 (1997).

[23] Y. Shimazaki, M. Mitsuishi, S. Ito and M. Yamamoto, Langmuir 14, 2768 (1998).

[24] A.C. Fou, O. Onitsuka, M. Ferreira, M.F. Rubner and B. R. Hsieh, J. Appl. Phys. 79(10), 7501 (1996).

[25] O. Onitsuka, A.C. Fou, M. Ferreira, B. R. Hsieh and M.F. Rubner, J. Appl. Phys. 80 (7), 4067 (1996).

[26] M. Raposo and O.N. Oliveira Jr., Thin Solid Films, 327/329, 739 (1998).

[27] M. Raposo and O.N. Oliveira Jr., "Hydrogen-bond control of polymer adsorption in layer-by-layer films", submitted.

[28] M. Raposo and O.N. Oliveira Jr., Adsorption of poly(omethoxyaniline) in layer-by-layer films, submitted.

[29] N. Tillman, A. Ulman and T. L. Penner, Langmuir 5, 101 (1989).

[30] J. Richer, L. Stolberg and J. Lipkowski, Langmuir 2, 630 (1986).

[31] R. Maoz and J. Sagiv, Langmuir 3, 1045, (1987).

[32] S.D. Evans, E. Urankar, A. Ulman and N. Ferris, J. Am. Chem. Soc. 113, 4121 (1991).

[33] N.G. Hoogeveen, M. A. Cohen-Stuart, G. J. Fleer and M.R. Böhmer, Langmuir 12, 3675 (1996).

[34] G. Decher, Y. Lvov and J. Schmitt, Thin Solid Films 244, 772 (1994).

[35] J.H. Cheung, W.B.Stockton and M. F. Rubner, Macromolecules 30, 2712 (1997).

[36] K. Ariga, Y. Lvov and T. Kunitake, J. Am. Chem. Soc. 119, 2224, (1997).

[37] Y. Lvov, S. Yamada and T. Kunitake, Thin Solid Films 300, 107 (1997).

[38] J. A. He, L. Samuelson, L. Li, J. Kumar and S. K. Tripathy, Langmuir 14, 1674 (1998).
[39] A. Laschewsky, E. Wischerhoff, M. Kauranen and A. Persoons, Macromolecules 30, 8304 (1997).

[40] Y. Lvov, K. Ariga, I. Ichinose and T. Kunitake, Langmuir 12, 3038 (1996).

[41] Y. Lvov, K. Ariga, M. Onda, I. Ichinose and T. Kunitake, Langmuir 13, 6195 (1997).

[42] G. J. Kellogg, A. M. Mayes, W. B. Stockton, M. Ferreira, M. F. Rubner, S.K. Satija, Langmuir 12, 5109 (1996).

[43] I. Ichinose, H. Tagawa, S. Mizuki, Y. Lvov and T. Kunitake, Langmuir 14, 187 (1998).

[44] E.S. Pagac, D. C. Prieve and R.D. Tilton, Langmuir 14, 2333 (1998).

[45] A. Wu, J. Lee and M.F. Rubner, Mat. Res. Soc. Symp. Proc. Vol-488, (Materials Research Society 1998), p.63.

[46] J. Kim, K.G. Chittibabu, M. J. Cazeca, W. Kim, J. Kumar and S.K. Tripathy, Mat. Res. Soc. Symp. Proc. Vol-488, (Materials Research Society 1998), p. 527.

[47] S. L. Clark, M. F. Montague and P. T. Hammond, Macromolecules 30, 7237 (1997).

[48] L.H.C. Mattoso, R. M. Faria, L.O.S. Bulhões and A.G. Macdiarmid, J. Polymer Science: Polymer Chemistry 322147 (1994).

[49] V. V. Tsukruk, V. N. Bliznynk, D. Visser, A. L. Campbell, T. J. Bunning and W. W. Adams, Macromolecules 30, 6615 (1997).

[50] G.J. Fleer and J. Lyklema, in Adsorption from solution at the solid/liquid interface, edited by G.D. Parfitt and C.H. Rochester, (Academic Press, 1983), p. 153.

[51] G.J. Fleer, Personal Communication.

[52] M.A. Awan, V.L. Dimonie, L.K. Filippov and M.S. ElAasser, Langmuir 13, 130 (1997).

[53] K. Lowack and C. A. Helm, Macromolecules 3, 823 (1998).

[54] Y. Kaniyama and J. Israelachvili, Macromolecules 25, 5081 (1992).

[55] I. Benjamim, H. Hong, Y. Avny, D. Davidov and R. Neumann, J. Mater. Chem. 8, 919 (1998).

[56] D. Yoo, S. S. Shiratori and M. F. Rubner, Macromolecules 31, 4309 (1998). 